\title{
Safety and efficacy of fluconazole treatment for Candida oesophagitis in AIDS
}

\author{
Antonio Gil, Paz Lavilla, Eulalia Valencia, Vicente Pintado, \\ Miguel L. Dupla, Munther A. Khamashta, Juan García-Puig and \\ Julio Ortiz-Vázquez
}

\section{Department of Internal Medicine, La Paz Hospital, Universidad Autónoma, Madrid, Spain}

\begin{abstract}
Summary: The efficacy and safety of fluconazole in the treatment of oesophageal candidiasis in patients with the acquired immunodeficiency syndrome (AIDS) was assessed in 36 patients. Fluconazole, $200 \mathrm{mg}$ orally, was given on the first day, followed by $100 \mathrm{mg}$ daily for 4 weeks. Clinical and mycological evaluation was performed in 31 patients at the end of treatment and 24 were also assessed after 8 weeks of starting treatment. In 1 patient fluconazole was discontinued, 5 patients were lost to follow-up and 6 patients died during the study.

Clinical and mycological cure was achieved in all patients; in 31 of 36 patients the clinical picture resolved within a week. The cure was confirmed in 27 patients by oesophagoscopy. Two patients relapsed 1 month after stopping fluconazole but the reinstitution of therapy achieved cure. Asymptomatic fungal oropharynx colonization was evident in about $\mathbf{4 0} \%$ of patients during treatment and follow-up period. Fluconazole was well tolerated by all patients but mild to moderate increase of liver enzymes values occurred in 16. Treatment had to be discontinued in 1 patient with hepatic tuberculosis because of severe liver function abnormalities, but their relation with the drug was uncertain.
\end{abstract}

Fluconazole is an effective and safe treatment of oesophageal candidiasis in AIDS patients.

\section{Introduction}

Oesophageal candidiasis occurs in about $90 \%$ of acquired immunodeficiency syndrome (AIDS) patients. ${ }^{1}$ The conventional but unsatisfactory treatment for Candida oesophagitis in AIDS was parenteral amphotericin in short-course therapy of 8-10 days ${ }^{2}$ despite the need for intravenous administration and marked drug toxicity. Ketoconazole has also been useful to treat oesophageal candidiasis and it has been proposed ${ }^{3}$ as the treatment of choice for this condition. Problems associated with ketoconazole include ketoconazole-resistant Candida strains, ${ }^{4}$ impaired gastrointestinal absorption secondary to the hyposecretory gastropathy that occurs in AIDS patients, ${ }^{5-7}$ the risk of hepatitis, ${ }^{8}$ the interaction with other drugs, particularly rifampicin, ${ }^{9}$ and the unavailability of an intravenous preparation.

The new triazole agents (fluconazole, itraconazole) may overcome some of these difficulties. ${ }^{10-12}$ Fluconazole is effective in treatment of deep and superficial Candida infections in

Supported in part by a grant from Caja de Madrid Correspondence: M.A. Khamashta, M.D., Rayne Institute, St Thomas' Hospital, London SE1 7EH, UK Accepted: 30 January 1991 both healthy and immunosuppressed rats. ${ }^{13,14}$ Oropharyngeal candidiasis in AIDS patients responds satisfactorily to fluconazole ${ }^{15}$ as may oesophageal candidiasis. ${ }^{16}$

We describe the results of treatment with fluconazole of oesophageal candidiasis in a series of AIDS patients.

\section{Materials and methods}

Thirty six AIDS patients of mean age 28 years ( 25 male and 11 female), diagnosed according to criteria established by the Centers for Disease Control, ${ }^{17}$ were studied. The most important risk factor for AIDS was illicit drug use (31 patients). In 15 the diagnosis of AIDS had been established in the preceding year (11 cases) or before ( 4 cases). Thirty one patients had a total of 38 human immunodeficiency virus (HIV)-associated opportunistic infections or neoplasias. Previous liver disease was documented in 6 patients and 15 patients had elevated liver enzymes values at entry. Twenty six patients were also receiving potentially hepatotoxic therapy including isoniazid and rifampicin (7 patients), sulphonamides (9), zidovudine (11) and benzodiazepines (15). 
Mean total lymphocyte count was $892 / \mu l$ (range 150 to $2,360 / \mu \mathrm{l}$ ) at entry with a mean CD4 + cells count of $170 / \mu l$ (range 0 to $795 / \mu \mathrm{l}$ ) and a CD4/CD8 ratio of 0.23 (range $0-1$ ).

\section{Treatment protocol}

All patients gave written informed consent and the study was approved by the Hospital Ethics Committee.

Endoscopic diagnosis of oesophageal candidiasis was required for inclusion of a patient in the study. Patients were excluded from study if: (i) aged less than 18 years; (ii) pregnant or breast feeding; (iii) had active liver disease with aspartate aminotransferase (ASAT), alanine aminotransferase (ALAT) and alkaline phosphatase (AP) values above three times the upper normal limits, and bilirubin higher than $51.3 \mu \mathrm{mol} / \mathrm{l}$; (iv) previous hypersensitivity to azole derivatives; (v) concomitant use of other antifungal or liver enzyme inducing drugs (barbiturates, coumarin anticoagulants, oral hypoglycaemics or cyclosporin) or (vi) administration of any other investigational agent.

Patients were treated for 4 weeks, and followedup for 8 weeks after starting treatment (Figure 1). Fluconazole, $200 \mathrm{mg}$ orally, was given on day one followed by $100 \mathrm{mg}$ daily for 4 weeks. Follow on prophylactic fluconazole, $200 \mathrm{mg}$ once weekly, was given in 22 patients. Clinical assessment was performed daily in patients admitted to the hospital and weekly for outpatients.

\section{Methods}

HIV antibody was determined by ELISA ${ }^{18}$ and Western Blot ${ }^{19}$ methods. Lymphocyte subset determinations were performed by indirect immunofluorescence method. ${ }^{20}$ Candida serology was performed by haemagglutination (Lab. Roche) and immunoprecipitation (Lab. Pasteur). A Fuji FP3 endoscope with a Fuji Mod K24 16R biopsy punch was used for oesophagoscopy. Three tissue samples were obtained; two of them were processed for pathological examination and the third was cultured for fungi in Sabouraud's dextrose agar medium with and without chloramphenicol. The culture was incubated at $30^{\circ} \mathrm{C}$ and $37^{\circ} \mathrm{C}$, and fungus identification was performed using the Vitek system. ${ }^{21}$ Patients were monitored closely for adverse reactions. Student $t$ test applying Yate's correction was used for statistical analysis.

\section{Results}

\section{Clinical course and mycological results}

Thirty six patients entered the study; 31 completed the treatment period and 24 the follow-up. Six patients died of a non-Candida opportunistic infection during the study, two in the treatment period. Postmortem examination in 4 revealed no evidence of candidiasis. Five patients were lost to follow-up and fluconazole was discontinued in 1 patient after
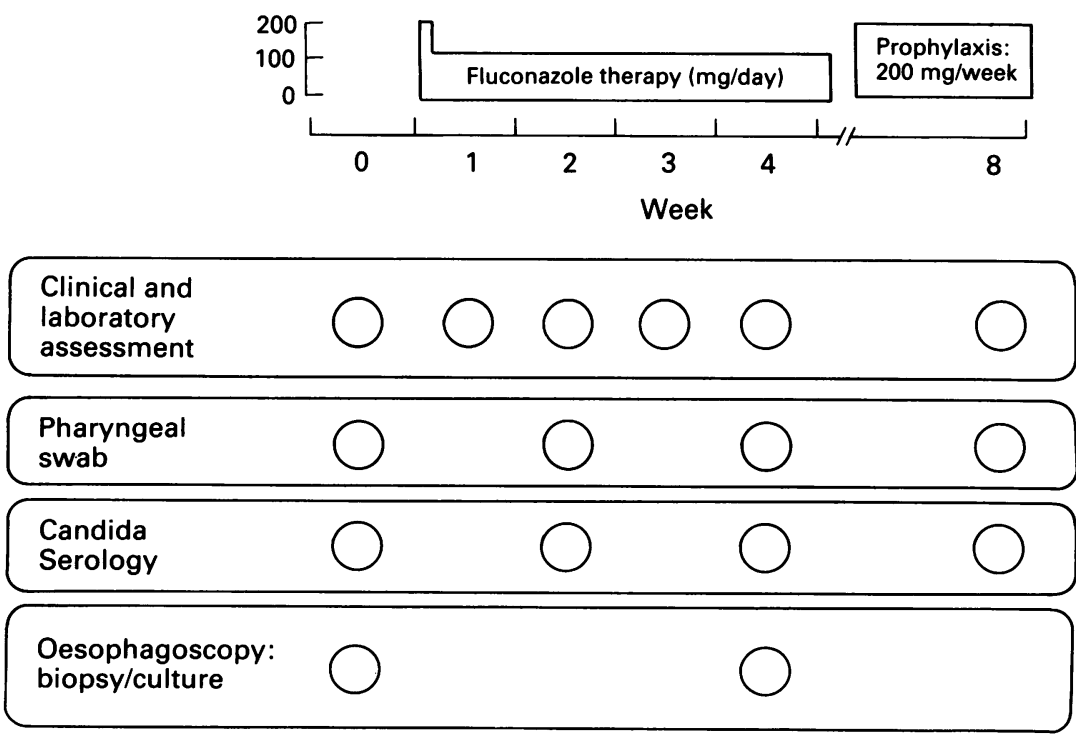

Figure 1 Study protocol. 
the first week because of deteriorating liver function tests. The results are summarized in Table $I$. All 36 patients had a positive pharyngeal swab for Candida albicans and oral thrush was present in $\mathbf{3 5}$ patients at entry. Nineteen patients complained of dysphagia. Oesophagoscopy showed typical appearance of candidiasis in 34 patients. Candida albicans was grown from biopsies in $30 / 32$ patients and identified histologically in $23 / 33$ oesophageal biopsies.

Clinical remission occurred in all 31 patients who completed the treatment. Signs or symptoms of oropharyngeal or oesophageal candidiasis were absent in all patients within 10 days of starting treatment, in 31 patients $(87 \%)$ within the first week. Oesophagoscopy was repeated in 27 patients 4 weeks after entry. Cure was confirmed by biopsy in 26 and by culture in 25 patients.

The results of fungal investigation from oropharyngeal swab are shown in Table II. After 2 weeks of therapy, cultures were negative in 19 patients (60\%). In the remaining patients, Candida albicans was the predominant isolated microorganism. After 4 weeks of treatment, there was still a predominance of Candida albicans, although other species and mixed fungal infections were also present. Candida albicans was present in oropharyngeal swab from 10 out of 24 patients who completed the follow-up; there were less than 5 colonies in all cases.

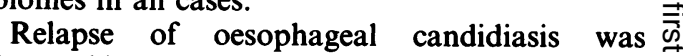
observed in 2 patients 1 month after completion of $\bar{C}$ fluconazole.

\section{Adverse reactions}

Fluconazole therapy was well tolerated by all patients and there was no haematological, renal, neurological, gastrointestinal or cutaneous toxicity. Increases in liver enzyme levels occurred in 17 patients, 13 during the first week and 4 during the second. These abnormalities were considered as mild (if liver enzymes were less than 3 times basal levels) in 15 patients, moderate (between 3 and 5 times) in 1 patient and severe (greater than 5 times basal levels) in another. In this latter patient, whose liver enzyme values were initially normal, fluconazole therapy was discontinued on the 7th day of treatment because of grossly abnormal liver function tests (AP $1640 \mathrm{U} / 1$, GGT $870 \mathrm{U} / \mathrm{l}$ ). A liver

Table I Clinical, endoscopic and mycological results

\begin{tabular}{llccc}
\hline & & \multicolumn{3}{c}{ Week } \\
& & $\begin{array}{c}0 \\
(n: 36)\end{array}$ & $\begin{array}{c}4 \\
(n: 31)\end{array}$ & $\begin{array}{c}8 \\
(n: 24)\end{array}$ \\
\hline Clinical picture: & Thrush & 35 & 0 & 2 \\
Pharyngeal swab: & Dysphagia & 19 & 0 & 2 \\
& Positive & 36 & 12 & 11 \\
Oesophagoscopy: & Negative & 0 & 16 & 13 \\
Oesophageal biopsy: & Diagnostic & 34 & 0 & 2 \\
& Mon-specific & 2 & 27 & 0 \\
Oesopis & 23 & 0 & 2 \\
& oesophagitis & 8 & 8 & 0 \\
& Normal & 2 & 18 & 0 \\
& C. albicans & 30 & 0 & 2 \\
& Negative & 2 & 25 & 0 \\
\hline
\end{tabular}

Table II Fungal colonization of oropharynx during and after fluconazole

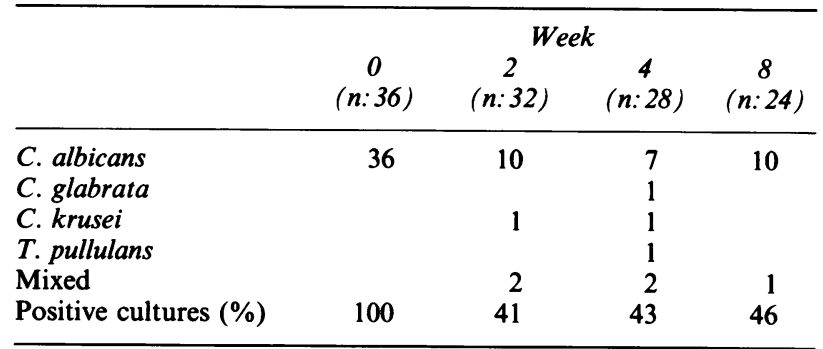


biopsy revealed Mycobacterium tuberculosis infection. Thus fluconazole toxicity was unlikely, but cannot be excluded. In the patients whose liver function tests became abnormal no changes in their fluconazole therapeutic regime were required. At follow-up liver function tests tended to return to normal. No correlation was found between liver enzyme abnormalities and the patients' age, sex, weight, previous liver disease, or abnormal liver enzyme values, ethanol intake, concomitant infections or drug treatment. A marked decrease in CD4 + cell count and the simultaneous administration of more than 3 drugs seemed to confer a higher risk of possible fluconazole-induced hepatotoxicity, although this was not statistically significant $(P<0.1)$.

\section{Discussion}

The present report is the most extensive one published as yet on fluconazole treatment for oesophageal candidiasis in AIDS patients. In all cases, the endoscopic findings, the histological picture and the mycological culture confirmed the efficacy of fluconazole. Clinical cure was rapid with symptoms of oesophageal involvement resolving within the first week of treatment in $87 \%$ of patients and in all patients after 10 days of therapy.

After successful fluconazole therapy asymptomatic fungal colonization of the oropharynx is commonly observed in AIDS patient ${ }^{22}$ and may cause recurrence of clinical illness. In our series (Table II) oropharyngeal colonization was present in $43 \%$ at the end of the treatment period and some of the most azole-resistant Candida species were recovered. The early appearance of relapses after completing fluconazole treatment in our initial 2 patients prompted us to devise a prophylactic regime. Oral fluconazole, $200 \mathrm{mg}$ once a week, was given to a further 22 patients and none presented relapses during the follow-up period. If this simple prophylactic regime proves to be effective in more extended series, this will represent an obvious advantage over existing preventive regimens for Candida oesophagitis. This regimen would increase patients' compliance and decrease the risk of interaction with drugs used for prophylaxis and treatment of other opportunistic infections in AIDS patients.

Fluconazole has shown little toxicity in experimental fungal infection models and clinical studies. ${ }^{23}$ Increases of liver enzymes ${ }^{24-26}$ have been reported during fluconazole therapy but in only 16 $(0.4 \%)$ of the first 4057 patients treated with fluconazole was it necessary to discontinue treatment due to significant hepatotoxicity (Pfizer Central Research - Data on file). In our series, an unexpectedly high incidence $(47 \%)$ of mild (15 patients) to moderate (1 patient) increase in liver enzymes values was observed. Several factors must be taken into account in considering the high incidence of hepatic abnormalities observed in our patients. A higher incidence of adverse drug effects has been reported to occur in AIDS patients. ${ }^{27}$ Moreover, most of our patients were in advanced stages of the disease as demonstrated by the severe decrease in CD4 lymphocyte count and the high mortality rate (6 out of 36) observed during the time of the study. Other opportunistic infections which might have involved the liver coexisted in 31 patients. Potentially hepatotoxic drugs were administered to 26 patients. Thus, patients in this series constitute a subgroup with a greater tendency to develop hepatic abnormalities than non-AIDS (or even HIV-positive with good general health status) patients with superficial mycoses who are not receiving other potentially hepatotoxic drugs and who usually receive smaller doses of fluconazole.

The rapidity of the clinical response in our treated patients as well as the results from other studies on AIDS patients with oropharyngeal candidiasis treated with lower doses $(50 \mathrm{mg} /$ day $)$ of fluconazole for shorter periods, ${ }^{28,29}$ suggests that with treatment for less than 4 weeks it might be possible to achieve cure in most patients.

We conclude that 4 weeks of fluconazole is a very effective treatment for oesophageal candidiasis in AIDS patients. Shorter treatment periods (i.e. 7-10 days) may be eventually efficacious. Further studies are necessary to establish the most effective follow-up prophylactic regimen for oesophageal candidiasis in AIDS. Such studies should also examine the potential for hepatotoxicity and predictive risk factors.

\section{References}

1. Klein, R.S., Harris, C.A., Small, C.B. et al. Oral candidiasis in high-risk patients as the initial manifestation of the acquired immunodeficiency syndrome. $N$ Engl $J$ Med 1984, 311: 354-358.

2. Tuazon, C.U. \& Labriola, A.M. Management of infectious and immunological complications of acquired immunodeficiency syndrome (AIDS). Current and future prospects. Drugs 1987, 33: 66-84.
3. Fazio, R.A., Wickremesinghe, P.C. \& Arsura, E.L. Ketoconazole treatment of Candida esophagitis - a prospective study of 12 cases. Am J Gastroenterol 1983, 78: 261-264.

4. Tavitian, A., Raufman, J.P., Rosenthal, L.E., Weber, J., Webber, C.A. \& Dincsoy, H.P. Ketoconazole resistant Candida esophagitis in patients with acquired immunodeficiency syndrome. Gastroenterology 1986, 90: 443-445. 
5. Lelawongs, P., Barone, J.A., Izzi, J.L. et al. Effect of food and gastric acidity on absorption of orally administered ketoconazole. Clin Pharm 1988, 7: 228-235.

6. Lake-Bakaar, G., Tom, W., Lake-Bakaar, D. et al. Gastropathy and ketoconazole malabsorption in the acquired immunodeficiency syndrome (AIDS). Ann Intern Med 1988, 109: 471-473

7. Lake-Bakaar, D., Beidas, S., El-Sakir, R., Iyer, S. \& Straus, E. Impaired gastric acid secretion in AIDS. Gastroenterology 1987, 92: 1488A.

8. Lewis, J.H., Zimmerman, H.J., Benson, G.D. \& Ishak, K.G. Hepatic injury associated with ketoconazole therapy. Gastroenterology 1984, 86: 503-513.

9. Engelhard, D., Stutman, H.R. \& Marks, M.I. Interaction of ketoconazole with rifampin and isoniazid. $N$ Engl $J$ Med 1984, 311: $1681-1683$.

10. Walsh, T.J. \& Pizzo, A. Treatment of systemic fungal infections: recent progress and current problems. Eur J Clin Microbiol Infect Dis 1988, 7: 460-475.

11. Saag, M.S. \& Dismukes, W.E. Azole antifungal agents: emphasis on new triazoles. Antimicrob Agents Chemother 1988, 32: 1-8.

12. Warnock, D.W. Itraconazole and fluconazole: new drugs for deep fungal infection. J Antimicrob Chemother 1989, 24: 275-280.

13. Troke, P.F. Efficacy of fluconazole in animal models of superficial and opportunistic systemic fungal infection. In: Fromtling, R.A. (ed.) Recent Trends in the Discovery, Development and Evaluation of Antifungal Agents. J.R. Prous Science Publishers SA, Barcelona, 1987, pp. 103-112.

14. Fisher, M.A., Shen, S.H., Haddad, J. \& Tarry, W.F. Comparison of in vivo activity of fluconazole with that of amphotericin B against Candida tropicalis, Candida glabrata and Candida krusei. Antimicrob Agents Chemother 1989, 33: 1443-446.

15. Dupont, B. \& Drohuet, E. Fluconazole in the management of oropharyngeal candidiasis in a predominantly HIV antibody positive group of patients. J Med Vet Mycol 1988, 26: 67-71

16. . Farrow, P.R., Brammer, K.W. \& Feczko, J.M. Fluconazole: a new treatment for oropharyngeal candidiasis in AIDS and malignancy. 27th ICAAC (Oct 4-7, New York) 1987; Abst 949.
17. Centers for Disease Control: Revision of the CDC surveillance case definition for acquired immunodeficiency syndrome. MMWR 1987, 36 (Suppl 1): 1S-15S.

18. Tsang, V.C.W., Peralta, J.M. \& Simons, A.R. Enzyme-linked immunoelectrotransfer blood techniques (EITB) for studying the specificities of antigen and antibodies separated by gel electrophoresis. Methods Enzymol 1983, 92: 347-349.

19. Brun-Vezinet, F., Rouzioux, C., Barre-Sinoussi, F. et al. Detection of IgG antibodies in patients with AIDS or lymphadenopathy syndrome. Lancet 1984, i: 1253-1256.

20. Kung, P.C., Goldstein, G., Reunherz, E.L. \& Schlossman, S.F. Monoclonal antibodies defining distinctive human $\mathrm{T}$ cell surface antigens. Science 1979, 206: 347-349.

21. Aldridge, C., Jones, P.W., Gibson, S.F. et al. Automated microbiological detection identification system. J Clin Microbiol 1977, 6: 406-413.

22. Meunier, F. Fluconazole treatment of fungal infections in the immunocompromised host. Semin Oncol 1990, 17 (Suppl 6) 19-23.

23. Tachibana, M., Noguchi, Y. \& Monro, A.M. Toxicology of fluconazole in experimental animals. In: Fromtling, R.A (ed.) Recent Trends in the Discovery, Development and Evaluation of Antifungal Agents. JR Prous Science Pub SA, Barcelona, 1987, pp. 93-102.

24. Van't Wout, J.W., Mattie, H. \& van Furth, R. A prospective study of the efficacy of fluconazole (UK-49858) against deep-seated fungal infections. $J$ Antimicrob Chemother 1988 , 21: $665-672$.

25. Galgiani, J.N. Fluconazole, a new antifungal agent. Ann Intern Med 1990, 113: 177-179.

26. Denning, D.W. \& Stevens, D.A. New drugs for systemic fungal infection. Br Med J 1989, 299: 407-408.

27. Gordin, F.M., Simon, G.L., Wofsky, C.B. \& Mills, J. Adverse reactions to trimethoprim-sulfametoxazole in patients witho the acquired immunodeficiency syndrome. Ann Intern Med 1984, 100: 495-499.

28. DeWit, S., Weerts, D., Goossens, H. \& Clumeck, N. Comic parison of fluconazole and ketoconazole for oropharyngea candidiasis in AIDS. Lancet 1989, i: 746-747.

29. Chave, J.P., Cajot, A., Bille, J. \& Glauser, M.P. Single dose therapy for oral candidiasis with fluconazole in HIV-infected adults: a pilot study. $J$ Infect Dis 1989, 159: 806-807. 